\section{Lobular Neoplasia of the Breast: Rethinking the Known}

One of the strengths of clinical practice guidelines is their dynamic nature. Rather than representing absolute truths, they reflect the best evidence available when they are produced. Periodic review is, therefore, mandatory. Given the prodigious outpouring of new data in oncology, NCCN has determined that guidelines must be reviewed at least annually to remain valid, with interim modifications for important information emerging within the cycle. This pursuit of change is at the core of guidelines utility: to provide a basis for physician and patient decision-making, they must be as up-to-date as the data.

For the most part, innovations-new technology advances, either in the diagnostic or therapeutic realms-lead to changes. Often these changes are incremental, such as replacing previously recommended adjuvant regimens with new combinations reported in well-conducted randomized trials. In some instances, new technologies can change the entire approach to a disease, as seen in how GIST tumors are managed now that responsiveness to imatinib mesylate and subsequent agents was demonstrated. In this case, GIST was separated from a group of sarcomas for which relatively similar care had been recommended.

In this issue, Andersen et al. address the current management of lobular neoplasia of the breast. As they note, for the past 20 years, lobular carcinoma in situ (LCIS) was considered a risk marker for subsequent breast cancer but not a malignant lesion itself. The therapeutic implications of this idea were that no further treatment other than biopsy was required, even if margins were positive. What Anderson et al. elegantly outline is that the basic concept of the disease is changing. The demonstration of a variant form of LCIS called pleiomorphic LCIS, the finding that pleiomorphic LCIS may be associated with the development of invasive lobular carcinoma, and the finding that some LCIS had previously been called DCIS (but now could be differentiated by E-cadherin expression) have all been reported in the past 10 to 15 years. In effect, these discoveries force us, and eventually guidelines developers, to re-conceptualize lobular neoplasia and examine how these new facets alter the basic approach to managing the disease. The lymphatic oncologists made similar adjustments as mantle cell lymphoma split off as a unique entity.

Our understanding of a specific tumor is made by conjoining diverse but re-enforcing observations so that we see a coherent picture of the natural history of the neoplastic process and can form a rational plan for intervening. The essence of science is the continued re-examination of our concepts in light of new data. When these new observations point to possible deficiencies in the underlying disease concept, new hypotheses can be generated and new studies launched to determine if the old paradigm must be modified. In some instances, this new orientation could lead to simplifying the approach. However, given the availability of ever more sophisticated laboratory technology in genetics and molecular chemistry, the odds are that we will be expanding our concepts of disease and the resultant approaches will entail increasing complexity in our scientific scaffolding. For guidelines developers, Einstein's dictum, "Everything should be made as simple as possible, but not simpler" must be a guiding principle.

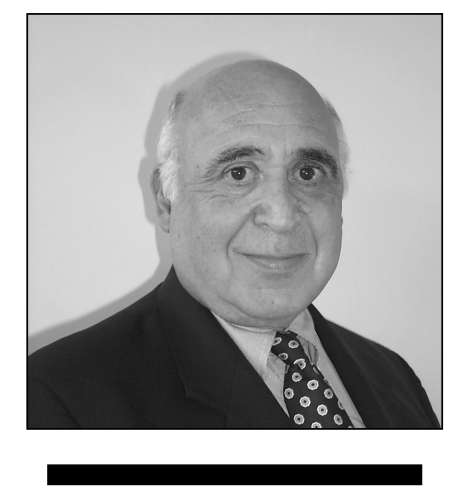

By Rodger J. Winn, MD

Rodger J. Winn is the Editorin-Chief of JNCCN. He is Clinical Consultant at the National Quality Forum, and his past positions include Associate Professor of Clinical Medicine at The University of Texas M. D. Anderson Cancer Center. Dr. Winn received his medical degree from Jefferson Medical College of Philadelphia. His postgraduate training includes an internship and residency at Jefferson Medical College, and he also completed a medical oncology fellowship at Memorial Sloan-Kettering Cancer Center in New York. He is board certified in internal medicine and holds subspecialty certification in oncology. 